\title{
Timing accuracy of the GEO 600 data acquisition system
}

\author{
K Kötter ${ }^{1}$, M Hewitson ${ }^{2}$ and H Ward ${ }^{2}$ \\ ${ }^{1}$ Max-Planck-Institute for Gravitational Physics (Albert-Einstein-Institute) and University of \\ Hannover, Callinstr. 38, D-30167 Hannover, Germany \\ ${ }^{2}$ Department of Physics and Astronomy, University of Glasgow, Glasgow G12 8QQ, \\ $\mathrm{UK}$ \\ E-mail: Karsten.Koetter@aei.mpg.de
}

Received 29 August 2003

Published 3 February 2004

Online at stacks.iop.org/CQG/21/S493 (DOI: 10.1088/0264-9381/21/5/016)

\begin{abstract}
This paper describes the tests done for validating the timing accuracy of the GEO 600 data acquisition system. Correct time stamping of the recorded data is required for a number of search algorithms for gravitational wave signals (coincidence analysis, targeted pulsar searches, etc). Tests on the current system determined the absolute timing offset to be $15.89 \mu$ s with a standard deviation of 63 ns. Both offset and jitter were measured against an external reference clock. Additional analysis of data recorded during the S1 data taking run was done to validate the timing accuracy during this period.
\end{abstract}

PACS numbers: $04.80 . \mathrm{Nn}, 95.55 . \mathrm{Ym}, 93.65 .+\mathrm{e}$

(Some figures in this article are in colour only in the electronic version)

\section{Introduction}

The GEO 600 data acquisition system (DAQS) [1] uses ICS110B [7] analogue-to-digital converter (ADC) boards operating at a sampling rate of $16384 \mathrm{~Hz}$ to record the signals of the GEO 600 [2-4] detector.

The timing of the data acquisition process is controlled by a GPS receiver in conjunction with an electronic circuit board that we will refer to as a 'timing card'. This board receives the timing information from the GPS unit and generates the signals required for driving the ADC board. The timing information provided by this hardware allows proper time stamping of the data. This is important because a number of search algorithms for gravitational wave signals rely on accurate time stamping of the data. To avoid any problems in a targeted search for a known pulsar, a timing offset of less than $10 \mu \mathrm{s}$ [8] is desirable. For burst signals the arrival time of the signal at the detectors of a worldwide network can be used to determine the direction of the source in the sky. The accuracy of this estimation is limited by the timing 


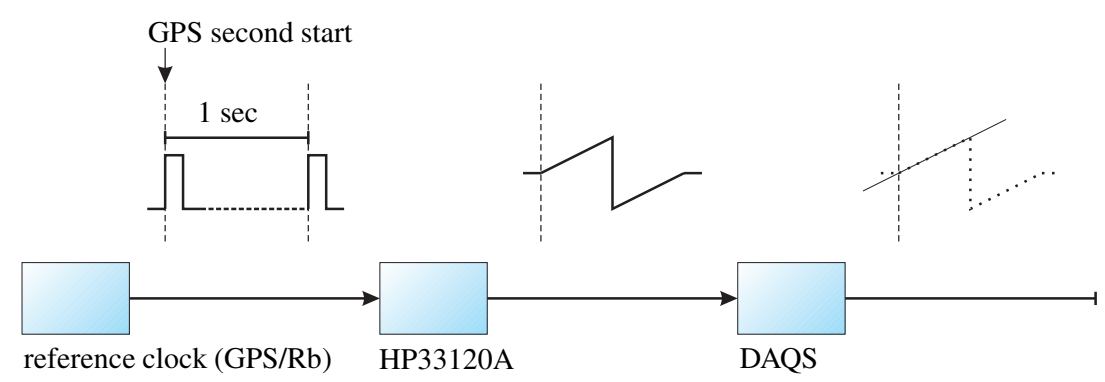

Figure 1. Set-up for the timing test: a reference timing unit was used to trigger a ramp signal from an HP33120A signal generator at the start of each GPS second. The signal was recorded in the DAQS and the start of the ramp signal determined by calculating the zero-crossing of a line fitted to the ramp.

offsets in the individual DAQ systems which need to be significantly smaller than the signal travel time between the detectors.

The ADC board employed in the GEO 600 DAQS has a gate delay of approximately 37 samples. This means that there is some delay between a signal connected to one of its inputs and the sampled output data. The major portion of this gate delay ( 34 samples) arises from the digital filter inherent to the ADC chip. The remainder of the measured delay is thought to arise from other clock delays in the circuits of the board. To account for this delay the software that controls the sampling process of the ICS110B removes the gate delay from the data by discarding the first 37 samples when the board is first initialized.

The data are recorded in $1 \mathrm{~s}$ segments, each synchronized to start at the beginning of a GPS second. This paper reports on analysis of the performance of the timing system by confirming the GPS second labels on the data segments and by measuring the residual offset and jitter of the start of each data segment against an external reference clock.

\section{Testing the system}

\subsection{Checking the GPS second labels on the data}

The question of whether or not the data are correctly time stamped at the $1 \mathrm{~s}$ scale was answered with a simple test: a signal was manually unplugged in the middle of a second using a DCF77 [6] clock as reference. It was then checked in the data whether the signal disappeared in the correct data segment. We observed that the time stamps on the data are correct.

\subsection{Measuring the residual offset and jitter against an external time reference}

To test the timing accuracy of the DAQ system to a high precision, we injected a test signal into one of the DAQ channels. A schematic of the test set-up is shown in figure 1. An external timing unit consisting of a rubidium atomic clock locked to a GPS receiver for long term stability was used to generate a trigger signal at the start of each GPS second. This signal was fed into an HP33120A signal generator that produced a digitally synthesized ramp with an amplitude of $1.7 \mathrm{~V}$ on each trigger event. The ramp signal was then injected into a DAQ channel.

A linear fit was made to the ramp as it appears in the data. By calculating the intersection of the fitted line with the zero level of the signal, the start of the ramp indicating the GPS second boundary could be determined to a precision much better than the sampling interval. Ideally the intersection point would be exactly at the beginning of each $1 \mathrm{~s}$ data segment. 


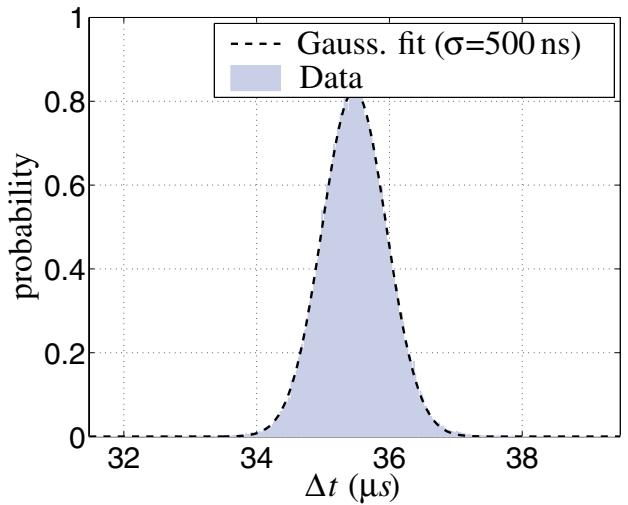

Figure 2. A histogram of the timing residuals of the current DAQ system taken over $24 \mathrm{~h}$. This is for an injected ramp length of $5 \mathrm{~ms}$. Although the mean of the offset is around $35 \mu \mathrm{s}$, this is partly due to the measurement method. Figure 3 extends this result to injected ramps of different lengths, resulting in a residual offset of $15.89 \mu$ s for an infinitely short ramp.

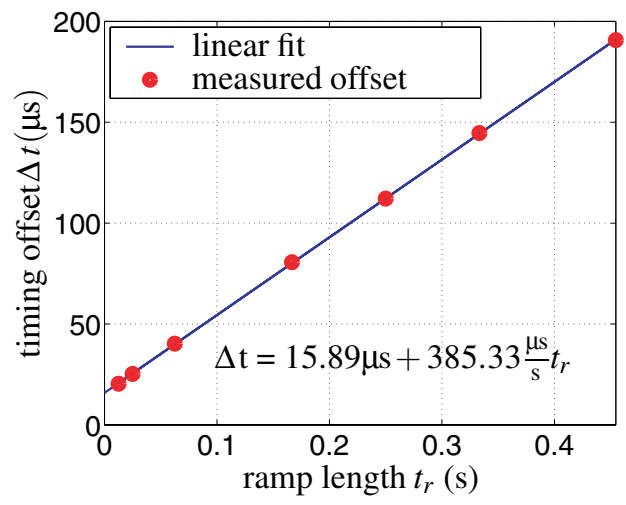

Figure 3. A plot of mean measured offset versus injected ramp length. A linear fit to the data is shown along with the equation of the best fit line.

However an offset $\Delta t$ between the intersection point and the beginning of the data segment was observed. A histogram of the offset measured once per second over a $24 \mathrm{~h}$ period can be seen in figure 2. A ramp length of $5 \mathrm{~ms}$ was used for this experiment. The distribution of the offsets is Gaussian with a standard deviation of $500 \mathrm{~ns}$. The mean value was $35.5 \mu \mathrm{s}$ and all measured values lay between $29.6 \mu \mathrm{s}$ and $40.2 \mu \mathrm{s}$.

We also observed that the measured offset and the jitter were both dependent on the length of the ramp signal that was used. The dependence of the offset on the ramp length is introduced by the digital signal generator used for the experiment. The starting point of the ramp signal generated by this device has a systematic time offset to the trigger signal. This offset grows with increasing ramp length. A shorter, steeper ramp makes the measurement less susceptible to errors due to amplitude noise leading to an error in the slope estimation: the steeper the ramp, the smaller the deviations of the measured zero-crossing for small fluctuations about the mean slope of the ramp. To separate the effects of our analysis from the timing offset and scatter inherent to the DAQ system, we repeated the above analysis with different ramp lengths. Figure 3 shows a plot of the mean measured offset versus injected 


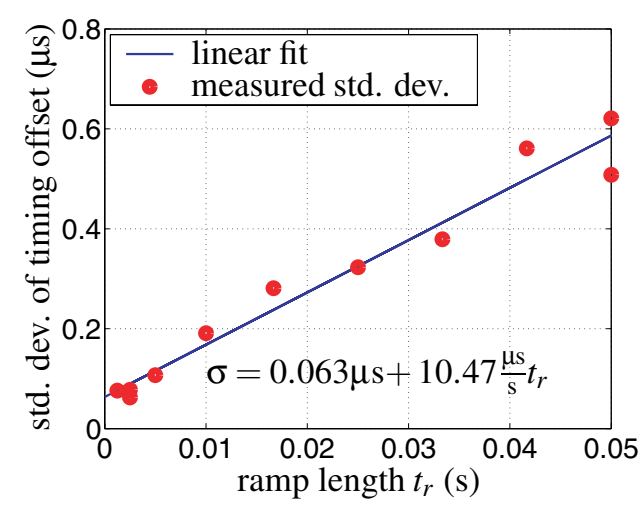

Figure 4. A plot of the standard deviation of each set of offset measurements against the length of the ramp signal used to make the measurements. A linear fit to the data is shown along with the equation of the best fit line. Each data point is the result of about 1500 measurements.

ramp length. A linear fit to the data allows us to extrapolate the value for an infinitely short ramp. From the fit we determine the residual timing offset of the DAQ system to be $15.89 \mu \mathrm{s}$ with the initial 37 samples gate delay removed. The offset is smaller than half the sample time $\left(\frac{1}{2} \frac{1}{16384 \mathrm{~Hz}} \approx 30.5 \mu \mathrm{s}\right)$, and therefore cannot be decreased by shifting the data by an integer number of samples. Algorithms exist that can shift the data by a fraction of a sample so that this known and constant $15.89 \mu$ s offset can be removed if required. This is not done by the DAQS software but left for posterior data conditioning.

Figure 4 shows a plot of the standard deviation of the offset measurements for each ramp length. Again we can extrapolate the value for an infinitely short ramp and see that the standard deviation in the measured offset due to timing fluctuations is $\sigma \approx 63 \mathrm{~ns}$.

\section{Problems found with the timing system during the tests}

\subsection{Timing across reboots}

The DAQS can be rebooted in two different ways. A hardware reboot (type I) means recycling the power to the full DAQ crate. A software reboot (type II) is performed using the interface software supplied with the processor board-here the processor board operating system is rebooted and the DAQ code is run from the start. During type II reboots, the power to the cards in the DAQ crate is maintained. We saw that a type II reboot causes a shift in the absolute timing offset of about $2300 \mu \mathrm{s}$. This value corresponds to the gate delay (see section 1) of 37 samples inherent to the ADC board: $\frac{37}{16384} \mathrm{~s} \approx 2258 \mu \mathrm{s}$.

The data buffer interrupt handler of the ICS110B ADC board does not behave in the same way as after a hardware reboot. Data are read from the ICS110B on board buffer every time the buffer-half-full interrupt fires. After a software reboot, the routine that waits for a buffer-half-full interrupt does not respond correctly on the first call of the function (although this is not the case on a hardware reboot). Calling the function a second time in the start-up procedure seems to work and there are no problems in all subsequent calls. Although this is not necessary on a hardware reboot, it has no adverse effects, so the routine is now rewritten to include a second call to this function in the start up section of the code that removes the gate delay. This fixes the problems seen with type II reboots. 


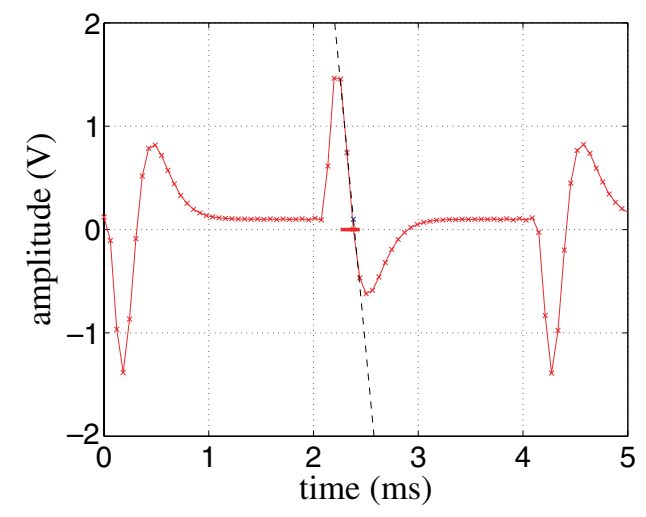

Figure 5. A close-up view of the calibration signal injected throughout S1. Also shown is the line fit to the falling slope of the signal at a particular time.

\subsection{GPS frequency lock servo}

We measured the timing offset after the DAQS hardware had been power cycled. It turned out that the timing offset deviates by up to $3 \mu$ s from the final value during the first 10 min after the GPS receiver was powered up. This effect is caused by the servo on the receiver that phase locks the internal oscillator of the device to the GPS signal. It takes approximately $10 \mathrm{~min}$ until the frequency of the internal oscillator settles to the correct value.

\section{S1 timing accuracy}

The ramp signal used for the test described above was not available during the $\mathrm{S} 1$ data run from 2002-08-23 15:00:00 to 2002-09-09 15:00:00 (UTC). To test the timing accuracy during that period, we used the calibration signal [5] for the detector output. This signal was suitable for such a test because the generator producing the signal was frequency locked to the GPS/Rb timing unit described in the previous section and the signal was recorded in the DAQS for the entire time of the S1 data run. This signal (see figure 5) was a square wave with a repetition rate of $244 \mathrm{~Hz}$ produced by an HP33120A signal generator, differentiated twice to give a set of spectral peaks.

Figure 5 shows a close-up view of this signal. Also shown is a slope fitted to a particular part of the signal. This fit was performed once every $15 \mathrm{~s}$ for the whole of the S1 data. Any change in the timing offset could be detected by tracking the zero-crossing of the fitted line. The upper graph of figure 6 shows the results of the analysis of all the S1 data. Two distinct variations in the measured zero-crossings can be seen in this figure: two periods where the values differ significantly (by about $2300 \mu \mathrm{s}$ ) and a general linear drift.

The timing of the DAQS does not drift in time as we know from the tests described above. The linear drift of the zero-crossing is caused by the signal generator producing the calibration signal. From the drift rate of the zero-crossing, one can see that the frequency of the signal is $243.999999938 \mathrm{~Hz}$ instead of the nominal value of $244 \mathrm{~Hz}$.

Because the calibration signal is not related to absolute GPS time, we cannot derive any absolute timing information from it. The large offset of more than $2000 \mu$ s displayed on the top of figure 6 showing the timing residuals for the entire S1 run is arbitrary-it just represents the point in the waveform that we chose to measure. This offset and the drift caused by the 

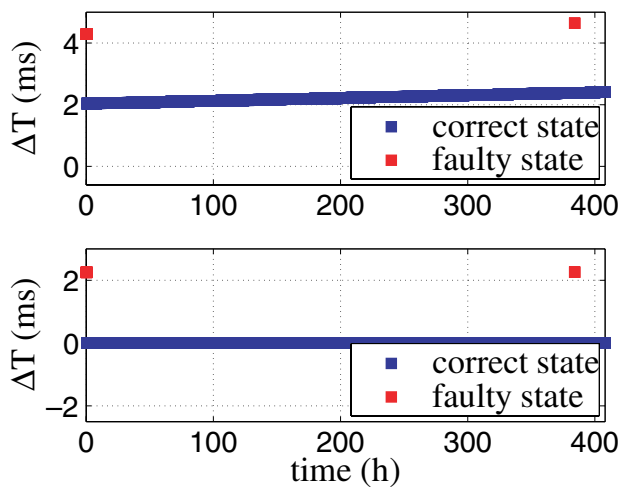

Figure 6. Time series showing the timing residuals for the whole S1 run. Zero time is at the start of the run on 2002-08-23 15:00:00 (UTC). Lower graph: timing residuals de-trended and offset removed. Values are around $0 \mathrm{~ms}$ in the correct state, and values around $2.3 \mathrm{~ms}$ indicate the two occurrences of a faulty state of the system in the entire S1 run.
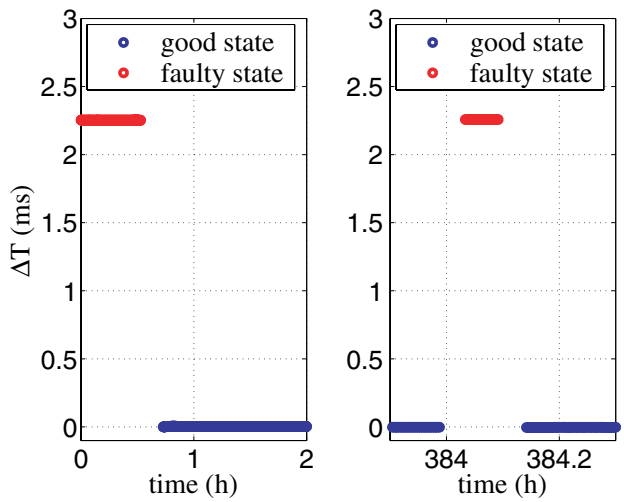

Figure 7. Close-up view of the periods when the system was in the faulty state. Again, the data are detrended and the offset removed. Note the different time scales in the two plots.

frequency offset of the signal generator were removed in the lower graph of figure 6 and subsequent plots.

From the previously described tests, we know that the timing offsets of the DAQ system are in the correct state after a hardware reboot. The two short periods where the measured zero-crossing differs significantly represent times when the DAQ system was rebooted via the software interface (type II). This type of rebooting was shown in section 2.2 to induce a state where the timing is offset by the gate delay of about $2300 \mu \mathrm{s}$. These times are highlighted in figure 7.

Figure 8 shows the timing residuals of the system in the correct state in more detail. One can see steps in the measured timing offset of about $10 \mu$ s that occur roughly every $70 \mathrm{~h}$. The times when the steps occur coincide with the times when the data segment used for fitting the slope to the calibration signal has to be moved because the drift of the calibration signal has accumulated to the time of one sample. This can be seen in the lower graph of figure 8 where a plot of the boundaries of the data segment used for the fitting process is shown. Moving the boundaries of this segment by one sample changes the slope and thus the zero-crossing of 

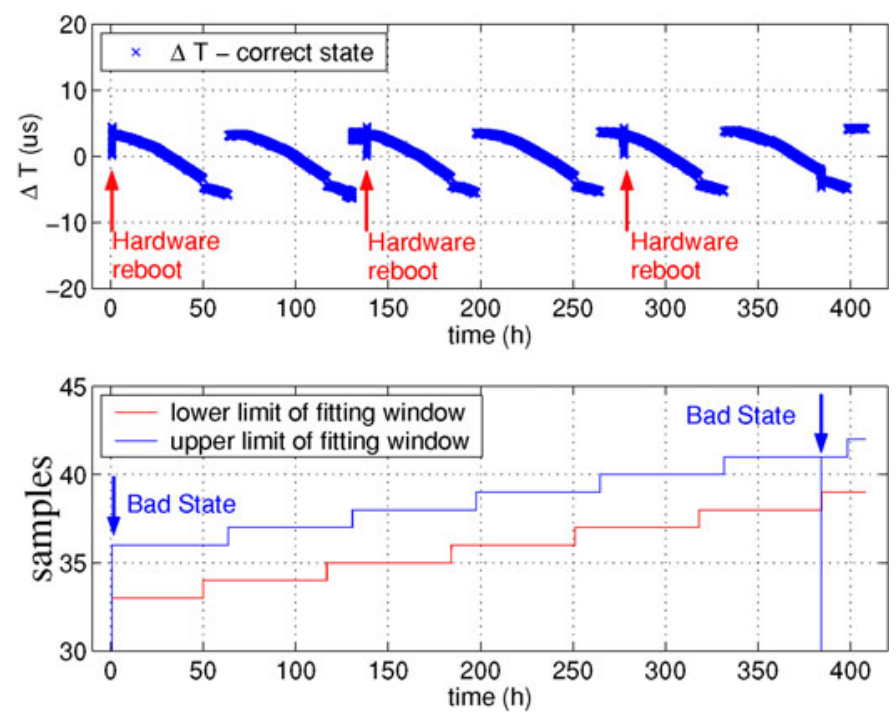

Figure 8. Upper graph: timing residuals when DAQ system was in good state. Steps in the values are due to changes in the fitting parameters. The lower graph shows the times when the parameters of the fitting window (start and stop sample) were changed. The vertical lines in the lower graph are the times when the system was in the bad state.

the fitted line, because the calibration signal used for the fitting process is only approximately linear.

The slow drift of the measured offset between the steps is also due to the non-linearity of the data segments being used for fitting: the slope of the fitted line changes as the non-linear signal segment drifts with respect to the sampling times.

The upper graph of figure 8 also shows three small vertical glitches that occur at times when the DAQ system was hardware rebooted (i.e. power cycled). These are caused by the servo of the GPS receiver as was explained in section 3.2.

\section{Summary}

We determined the data time stamping of the current GEO 600 DAQ system to have an absolute offset of around $+16 \mu \mathrm{s}$ which arises from the residual gate delay of the ADC board that cannot be compensated by full sample shifts of the data. If required this known and constant offset can be removed in posterior data conditioning.

We identified two periods (2002-08-23 15:00:00 to 2002-08-23 15:32:00 and 2002-09-08 15:01:00 to 2002-09-08 15:06:00) during the $S 1$ run where the time stamping of the data was offset from the nominal value by $\sim 2300 \mu \mathrm{s}$. The cause of these offsets was software rebooting of the DAQ system. In the future software reboots can be performed after an additional fix to the DAQ code. We were able to see the effect on the time stamping when the GPS receiver is powered down and back up again: excess timing fluctuations for about $10 \mathrm{~min}$ after the power is restored to the GPS receiver have been observed. In the good state (prevailing over $99.8 \%$ of the data run), we see (figure 4) that the time stamping of the data has a standard deviation of about $60 \mathrm{~ns}$. 


\section{Acknowledgments}

The authors acknowledge the support of the Land Niedersachsen (State of Lower Saxony) and the Particle Physics and Astronomy Research Council, UK.

\section{References}

[1] Koetter K et al 2002 Data acquisition and detector characterization of GEO 600 Class. Quantum Grav. 19 1399-1407

[2] Danzmann K et al 1994 GEO 600_-Proposal for a $600 \mathrm{~m}$ laser-interferometric gravitational wave antenna (MPQ Garching)

[3] Willke B et al 2002 The GEO 600 gravitational wave detector Class. Quantum Grav. 19 1377-87

[4] Hewitson M et al 2003 A report on the status of the GEO 600 gravitational wave detector Class. Quantum Grav. 20 S581

[5] Hewitson M et al 2003 Calibration of GEO 600 for the S1 science run Class. Quantum Grav. 20 S885

[6] Web page DCF77-Signal der Physikalisch Technischen Bundesanstalt http://www.dcf77.de/

[7] Web page Interactive Circuits and Systems Ltd http://www.ics-ltd.com/

[8] Allen B 2003 Private communications 